\section{P162 INCREASING TRENDS OF INFECTIOUS SYPHILIS IN WOMEN OF CHILDBEARING AGE IN AUSTRALIA}

'B Hengel*, 'L Causer, ${ }^{2} \mathrm{~A}$ Bright, ${ }^{1} \mathrm{H}$ McManus, 'S McGregor, 'B Donovan, ${ }^{3} \mathrm{~J}$ Ward, ${ }^{1} R$ Guy. ${ }^{1}$ Kirby Institute, UNSW, Sydney, Australia; ${ }^{2}$ Department of Health, Australian Government, Canberra, Australia; ${ }^{3}$ UQ Poche Centre for Indigenous Health, The University of Queensland, St Lucia, Australia

10.1136/sextrans-2021-sti.264

Background Syphilis in pregnancy can have devastating consequences with over half of untreated infections leading to serious complications. In Australia, an outbreak was detected in remote Indigenous communities in 2011, with recent increases in non-Indigenous heterosexuals. To understand the distribution and impact of these increases, we analysed notification trends in women of childbearing age.

Methods National infectious syphilis notification data (20112018) for women aged 15-44 years were used to calculate notification rates per 100,000 by Indigenous status, age, remoteness and year, and congenital syphilis cases per 100,000 live births by Indigenous status and year. We determined trends in notification rates using Poisson regression.

Results Between 2011-2018, there were 1391 and 909 notifications in Indigenous vs non-Indigenous women, with the rate 31 times higher (188 vs 6 per 100,000) in 2018, respectively. For Indigenous women, the highest rate was among women living in remote areas (522 per 100,000 in 2018) with increases in remote (174\%), regional (808\%) and urban areas $(475 \%), \mathrm{p}<0.001$; and by age group the highest rate was in $15-24$ year olds (214 per 100,000 in 2018), with increases in all age groups $(130 \%-971 \%) \mathrm{p}<0.001$. For non-Indigenous women, the highest rate was in urban areas (6 per 100,000 in 2018 ) with increases in urban (474\%), and regional areas $(475 \%)(\mathrm{p}<0.001)$; and by age group the highest rate was in 25-34 year olds (7 per 100,000 in 2018), with increases in all age groups $(383-541 \%)(\mathrm{p}<0.001)$. In 2018 , congenital syphilis rates were 14 times higher (19.6 vs 1.4 per 100,000 live births) in Indigenous women vs non-Indigenous women, respectively.

Conclusion Rates of infectious syphilis are increasing for Indigenous and non-Indigenous women of child-bearing age, with the greatest burden of disease in Indigenous women living in remote areas. Responses need to be delivered with further potency to control syphilis in Australia.

\section{P164 THE EPIDEMIOLOGY OF HERPES SIMPLEX VIRUS TYPE 2 IN ASIA: A SYSTEMATIC REVIEW, META-ANALYSES, AND META-REGRESSIONS}

1,2S AlMukdad*, 1,2M Harfouche, ${ }^{1} \mathrm{~A}$ Wettstein, 1,2,3 $\mathrm{L}$ Abu-Raddad. ${ }^{1}$ Infectious Disease Epidemiology Group, Weill Cornell Medicine-Qatar, Cornell University, Qatar Foundation Education City, Doha, Qatar; ${ }^{2}$ World Health Organization Collaborating Centre for Disease Epidemiology Analytics on HIVIAIDS, Sexually Transmitted Infections, and Viral Hepatitis, Weill Cornell Medicine-Qatar, Cornell University, Qatar Foundation - Education City, Qatar; ${ }^{3}$ Department of Population Health Sciences, Weill Cornell Medicine, Cornell University, USA

10.1136/sextrans-2021-sti.265

Background Herpes simplex virus type 2 (HSV-2) infection is a prevalent sexually transmitted infection worldwide. This systematic review aimed at characterizing HSV-2 epidemiology in Asia.

Methods HSV-2 publications were systematically reviewed. Findings were reported according to PRIMA guidelines.
Pooled measures and associations were assessed using randomeffect meta-analyses and meta-regressions.

Results From 173 relevant publications, 340 overall outcome measures and 729 stratified measures were extracted. Pooled mean HSV-2 seroprevalence was $12.1 \% \quad(95 \%$ confidence interval (CI): 11.0-13.2\%) among general populations, $23.6 \%$ (95\% CI: 20.9-26.3\%) among men who have sex with men and transgender people, $46.0 \%$ (95\% CI: 39.2-52.9\%) among HIV positive individuals and individuals in HIV discordant couples, and 62.2\% (95\% CI: 58.9-65.6\%) among female sex workers. Among general populations, pooled mean seroprevalence increased gradually from 4.7\% (95\% CI: 3.3-6.3\%) in $<20$ years-old individuals to reach $26.6 \%$ (95\% CI: 19.2 $34.7 \%$ ) in $>60$ years-old individuals. Compared to women, men had a 0.60 (95\% CI: 54.0-67.0) lower seroprevalence. Seroprevalence declined by 0.98 -fold (95\% CI: $0.97-0.99$ ) per year in the last three decades. Pooled mean proportions of HSV-2 detection in genital ulcer disease (GUD) and in genital herpes were $48.2 \%$ (95\% CI: $34.9-61.6 \%)$ and 75.9\% (95\% CI: $68.3-82.8 \%)$, respectively.

Conclusion In Asia, approximately 1 in 10 individuals is infected with HSV-2, but seroprevalence is declining by $2 \%$ per year. HSV-2 persists as the cause of nearly $50 \%$ of GUD cases and $75 \%$ of genital herpes cases.

\section{P165 EVALUATION OF NOVEL TREPONEMA PALLIDUM RECOMBINANT ANTIGENS FOR SYPHILIS DIAGNOSIS}

${ }^{1} \mathrm{E}$ Romeis*, ${ }^{1} \mathrm{~A}$ Haynes, ${ }^{1} \mathrm{~A}$ Phan, ${ }^{2,3} \mathrm{~K}$ Konda, ${ }^{2} \mathrm{~S}$ Vargas, ${ }^{2} \mathrm{M}$ Eguiluz, ${ }^{2} \mathrm{C}$ Caceres, ${ }^{3} \mathrm{~J}$ Klausner, 'L Giacani. 'University of Washington, Seattle, USA; ${ }^{2}$ Universidad Peruana Cayetano-Heredia, Lima, Peru; ${ }^{3}$ University of California Los Angeles, Los Angeles, USA

\subsection{6/sextrans-2021-sti.266}

Background Syphilis, caused by the spirochete Treponema pallidum subsp. pallidum (T. pallidum), continues to be a significant global health concern. The diagnosis of syphilis mainly relies on assessing clinical manifestations and performing serologic testing to detect treponemal and non-treponemal antibodies to discriminate between past and present infection and to evaluate response to treatment. Limitations, however, include that no test can discriminate among syphilis stages, and sensitivity is low during the very early stages of infection. New diagnostics could circumvent some of these deficiencies.

Methods To this end, we developed a novel array of T. pallidum recombinant proteins to identify seroreactive proteins and compare their performance to that of antigens commonly used in treponemal tests such as the 17 and $47 \mathrm{kDa}$ lipoproteins ( $\mathrm{Tp} 0435$ and $\mathrm{Tp} 0574$, respectively). Antigens were applied to the array, then reactivity was assessed via indirect enzyme-linked immunosorbent assay (ELISA). For this analysis, we used sera samples from 124 patients collected at various timepoints $(0$ months, 3 months, and 6 months). Samples from all three timepoints were available from 29 patients.

Results The control antigens demonstrated the highest reactivity against the sera. Among the antigens tested so far Tp0954, a putative adhesin of the syphilis agent, exhibited seroreactivity similar to that of the controls.

Conclusion Our data support Tp0954 as a possible candidate to improve the performance of currently available treponemal tests. 